\section{Use of US Veterans Affairs Administrative Databases to Study Outcomes in Patients with Rheumatoid Arthritis}

\section{To the Editor:}

In a recent issue of The Journal, Khurana, et $\mathrm{l}^{1}$ report on the increased risk of lung cancer in patients with rheumatoid arthritis (RA) using administrative data from the Veterans Integrated Service Networks (VISN). The methodology and conclusions of the study raise several issues. The first is the definition of the diagnoses of RA and lung cancer used in the study. The authors state that the diagnosis of RA was based on International Classification of Diseases (ICD-9) coding of 714.0 in the computerized records without any other qualifying criteria. Published data show that an ICD-9 code for RA when combined with the receipt of a disease-modifying agent (DMARD) in the Veterans Affairs (VA) administrative databases has a sensitivity of $76 \%-88 \%$ and a specificity of $83 \%-97 \%{ }^{2}$. Thus, use of the 714.0 code without additional criteria brings into question the accuracy of RA diagnosis in the database. More important, there is no information provided about criteria for the diagnosis of lung cancer in the database, which formed the basis for the definition of cases and controls. ICD-9 codes for lung cancer are numerous and range from 162.0 to 162.9 ; it is unclear which or whether one or more of these were used. The lack of such well defined criteria makes it likely that cases of RA and lung cancer were misclassified, raising questions about the validity of the results.

Second, the use of a tumor necrosis factor- $\alpha$ (TNF- $\alpha$ ) antagonist was not included as a variable when assessing the risk of lung cancer in the RA cohort. The reason for this as stated by the authors is that during the study period (October 1998 to June 2004), use of the TNF antagonists was not very common and that the duration of therapy was insufficient to influence the development of malignancy. Etanercept and infliximab were approved for RA in November 1998 and November 1999, respectively, and their use within the VA system was widespread during the study period. Our data from the national administrative VA databases show that 9377 patients with RA were receiving a TNF antagonist between October 1, 1998, and September 30, 2006. We defined RA in the database using an algorithm validated by Singh, et $a l^{2}$. Thus the diagnosis of RA required both (1) occurrence of an ICD-9-CM code for RA on at least one occasion in either the inpatient or outpatient record, and (2) the receipt of a prescription for a DMARD on at least one occasion. The following ICD-9-CM codes were defined as coding for RA: RA (714.0), Felty's syndrome (714.1), RA with visceral or systemic involvement (714.2), or rheumatoid lung (714.81) (unpublished data).

Third, the study suggested that lung cancer risk was significantly increased in Caucasians and African Americans with RA (Caucasians OR 1.44, 95\% CI 1.20-1.73, p < 0.01; African Americans OR 1.46, 95\% CI $1.05-2.02, \mathrm{p}=0.02)$. However, the race variable as it is recorded in the VA database has been shown to correlate poorly with patient self-report ${ }^{3}$. Also, there is a large number of unknown race recorded in the VA database. In our data, $21 \%$ (9953) of 47,454 patients who met our inclusion criteria for RA (as described above) were of unknown race, making interpretation of the effect of race on outcome unreliable (unpublished data). Given the significant number of patients of unknown race in Khurana's study, this finding of increased lung cancer risk in Caucasians and African Americans with RA is questionable.

In summary, Khurana, et al are to be commended for using a large VA database to address a clinically relevant question in an RA population. However, although the authors claim that their use of a computerized diagnosis database with ICD-9 coding methods decreased misclassification, this may not be true because of the reasons described above. The use of tighter definitions for RA and lung cancer and inclusion of TNF antagonist use as a covariate in the regression analysis would have enhanced the quality and validity of the findings.

PRABHA RANGANATHAN, MD, MS; ANGELIQUE L. ZERINGUE, MS JAY R. McDONALD, MD, St. Louis Veterans Affairs Medical Center, and Washington University, St. Louis, Missouri, USA. Address reprint requests to Dr. P. Ranganathan, Division of Rheumatology, Washington University School of Medicine, 660 S. Euclid Avenue, Campus Box 8045, St. Louis, MO 63110. E-mail: prangana@im.wustl.edu

\section{REFERENCES}

1. Khurana R, Wolf R, Berney S, Caldito G, Hayat S, Berney SM Risk of development of lung cancer is increased in patients with rheumatoid arthritis: a large case control study in US veterans. J Rheumatol 2008;35:1704-8.

2. Singh JA, Holmgren AR, Noorbaloochi S. Accuracy of Veterans Administration databases for a diagnosis of rheumatoid arthritis. Arthritis Rheum 2004;51:952-7.

3. Kressin NR, Chang BH, Hendricks A, Kazis LE. Agreement between administrative data and patients' self-reports of race/ ethnicity. Am J Public Health 2003;93:1734-9.

J Rheumatol 2009;36:5;doi:10.3899/jrheum.081055 